\title{
Change of physical and chemical parameters of the liquid binary systems by alternating impulses of pressure
}

\section{Iryna Dubovkina}

\section{Institute of Engineering Thermophysics of National Academy of Sciences of Ukraine, Kyiv, Ukraine}

Keywords:

Water

Ethanol

Mixture

Pressure

Impulse

\section{Article history:}

Received 02.12.2016

Received in revised

form 14.02.2017

Accepted 30.03.2017

Corresponding

author:

Iryna Dubovkina

E-mail:

dubovkinai@ukr.net

DOI: $10.24263 / 2304-$

974X-2017-6-1-16

\section{Abstract}

Introduction. It were conducted the researches with a purpose to determine the impact of non-reagent method, namely alternating impulses of pressure on the parameters of water-ethanol mixtures in a wide range of concentrations.

Materials and methods. The methods of direct potentiometry (ionometry) were used for the researches. Experimental investigations of liquid samples were carried out with use laboratory measurement devices: $\mathrm{pH}$-metermillivoltmeter pH-150 M and oximeter EZODO PDO-408.

Results and discussion. At influence of alternating impulses of pressure in liquid binary systems such as water systems and water-ethanol mixtures occurs intensive deoxygenating, decrease in quantity of the dissolved oxygen in water and water-ethanol mixtures in comparison with the initial maintenance occurs practically on $50-55 \%$.

During researches increases $\mathrm{pH}$ of the distilled water on $13 \%$ have been established, thus the hydrogen potential of the water prepared on technology of the distillery has raised on $14-14,5 \%$.

A value of redox potential in the course of processing by alternating impulses of pressure depending on processing time decreases on $20-60 \%$.

The change of physical and chemical properties and parameters of water systems has been established at processing application high-frequency oscillation which it is possible to explain change of reactionary ability, owing to initiation of carrying over of a proton in associated liquids such as water, aliphatic alcohols, water-ethanol mixtures with different percentage of ethanol and formation of a grid of hydrogen bonds which in turn influences the structural organisation and a structure.

Conclusions. A water and water-ethanol mixtures treatment by nonchemical reagentless method in rotarypulsating devices can greatly reduce the duration of the process of mixing mode, reduce power consumption, increase capacity and replace the batch process for the continuous mixing. 


\section{Introduction}

Nowadays one of the important problems that are of interest to the scientific community is to develop innovative nonstandard products and technologies that meet modern international standards of quality and safety.

Actual to solve this problem is to use low-cost methods that require cost-effective investment and allowing the use of existing reserves to reduce specific energy consumption of existing equipment due to the intensification of technological processes.

The sustainable development of modern food enterprises and food processing plants is impossible without the introduction of high-tech and energy-efficient production processes.

During the realisation of the processes which associated with requirement of uniform mixing and distribution of different phases in a liquid basis of great value gets possibility of forecasting of reaction of such systems depending on a different sort of external periodic power or energy influences.

Water and alcohol is sufficient difficult associate systems, which are sensitive to the smallest amount power influences. Formation of water-alcoholic mixtures is the process of mixing of water and alcohol.

In pure clean water and in the diluted solutions there is a continuous three-dimensional grid of hydrogen bonds, it proves to be true many researches and mathematical experiments $[1,2]$.

Inevitability of research of the structural organization of water and liquid binary systems is caused by their unique properties, and also exclusive value in the live and lifeless nature, a science and the technician, modern technologies.

The founding of principles and conditions of formation of structure and intermolecular hydrogen bonds gives the opportunity to change purposefully character and speed of many physical and chemical processes which take place in such liquid associated systems. Besides, physical and chemical parameters and properties depend on structural transformations and bonds which can create between molecules, for example hydrogen bonds.

Liquid binary systems, specifically aqueous-alcoholic such as mixtures of water and aliphatic alcohol (ethanol, methanol etc.), are enough complicated objects for researches as belong to open systems, and can exchange with environment not only energy, but also substance. At the same time it is metastable, systems which have the certain structural organization and structure which properties and parameters depend on many factors which are insufficiently studied.

One of the ways to improve the quality and safety of products derived benefits have processing technology using a reagent-free non chemical methods, modes and different devices of physical impacts and influences, without the use of chemical materials and substances.

\section{Analysis of scientific works}

During the past decade, there has been considerable investigation of the many alternative technological methods of treatment liquids.

Non-reagent methods of water and liquid binary systems treatment include:

- Acoustic treatment: ultrasonic treatment, sound treatment;

- The electromagnetic pulse effect of the low-frequency field;

- Cavitation processing; 
- Emitting treatment: ultraviolet, ionizing, infrared etc.

- Hydrodynamic effects.

In recent years researches and technologists have turned their attention to employment of power sound and ultrasound in processing.

Among available technologies, ultrasound technology has a significant potential to produce good-quality, healthful, delicious, and affordable convenience food products and different drinks [3]. The numerous applications of ultrasound, the approach are used in the field of water treatment. Ultrasonic treatment in a liquid leads to the acoustic cavitation phenomenon such as formation, growth, and collapse of bubbles (cavitation), accompanied by generation of local high temperature, pressure, and reactive radical species $\left({ }^{\circ} \mathrm{OH}\right.$, $\left.{ }^{\circ} \mathrm{OOH}\right)$ with thermal dissociation of water and oxygen [4].

Recently there has been considerable investigation of the electromagnetic pulse effect of the low-frequency field on water or on the behaviour of aqueous solutions. The physicochemical properties and parameters of water, such as: oxidation-reduction potential, $\mathrm{pH}$ value, dissolved oxygen and act. may be tainted by the magnetic and electromagnetic fields. These changes depend on the field intensity and frequency. Although intensive research, the mechanisms by which electromagnetic fields act on water are still a controversial issue [5]. Extremely low frequency electromagnetic fields have significant and lasting effects on liquid water [6].

The majority of theories explain effect of magnetic processing of water magnetic field action on there is at water ions of salts which are exposed to polarisation and deformation [7]. As main and key parameters of devices for processing of water by a magnetic field intensity of a magnetic field, time of stay of water serve in an active zone of a magnetic field, frequency rate and periodicity of influence of a field on water, speed of a stream of water in the device [8].

One of the innovate technologies that was used for improvement of water treatment process is application of cavitation processing. By definition cavitation consists in formation of ruptures of sites of a liquid (small vials), under the influence of the sharp changes of pressure caused by movement of a liquid.

Cavitation is the phenomena of the formation, growth and collapse of microbubbles or cavities occurring in extremely small interval of time (milliseconds) in a liquid [9]. Cavitation can be used as the working tool for the organisation of different technological processes, for example for: clarifications and processing's of surfaces, hashing of multiphase streams (a liquid - a liquid, gas - a liquid, firm particles - a liquid etc.), activation of chemical reactions, structuring and is final, in technologies of clarification and water disinfecting. In the conditions of cavitation hydroxyl $\left(\mathrm{OH}^{\circ}\right)$ and hydrogen $\left(\mathrm{H}^{\circ}\right)$ radicals would be formed by thermal dissociation of water and oxygen [10].

Emitting treatment: ultraviolet, ionizing, infrared is very perspective nowadays.

A number of alternative methods are commercially available for the removal of microbiological pollutants and some chemical contaminants from water sources. Conventionally, the ultraviolet spectrum is divided into three discrete sections:

- Ultraviolet A (320-400 nm);

- Ultraviolet B (280-320 nm);

- Ultraviolet C (less than $280 \mathrm{~nm}$ ).

Exposure to ultraviolet light can result in the formation of a range of photoproducts whose distribution and relative yields depend on the wavelength and intensity of incident radiation. 


\section{— Processes and Equipment of Food Productions -}

Infrared treatment of water used for micro-organism inactivation and structural transformation by the infrared radiation. But infrared laser water treatment apparatus is limited, however, by the energy consumption and cost required activating water.

Photocatalysis has great potential as an alternative water treatment method due to possibility to remove by-product precursors. This process also ensures the public health safety of drinking water due to its ability to inactivate micro-organisms and to change physical and chemical parameters of water.

Photocatalytic processes are divided into:

- homogeneous photocatalytic oxidation, e.g. ultraviolet/hydrogen peroxide

- heterogeneous photocatalytic oxidation, such as ultraviolet/semiconductor photocatalysis [11].

Hydrodynamic effects occur at water and water binary systems and mixtures treatment by physical and mechanical methods.

The method of discrete-pulsed input of energy (DPIE) is one of physical methods which can influence on structural transformations in complex liquid systems on micro- and nano- level and gives possibility to initiate physical and chemical changes in these systems [12].

The fundamental nature of a DPIE method consists in that preliminary permanently entered and any rank the energy distributed in working volume to accumulate in locally disconnected discrete points of system and further pulse to realise for achievement of necessary physical effects: forcing and dumping of pressure, adiabatic boiling, hydraulic blow, shock waves of pressure or depression, pressure of shift, local turbulence, cavitation effects.

Spatial and time concentration of energy gives the possibility to receive the big capacity of pulse power action, to liberate internal energy of substance, to make active processes which occur at microlevel also.

The method of discrete-pulsed input of energy is divided into such effects and mechanisms:

- effects which associated with acceleration of movement of a continuous phase;

- influence of pressure of shift;

- cavitations mechanisms;

- the mechanism of explosive boiling;

- collective effects in assembly of vials;

- indignation of an interphase surface in gas-liquid bubbly medium.

- action of alternating impulses of pressure.

Development of different microliquid devices for some last decades has caused growth of interest to microscale streams. Rotary pulse apparatus are characterised by small enough sizes of width of channels which gives the chance to consider them as microchannels with effects of slippage a stream on walls.

A number of heat and mass technological processes (structuring, crushing, dispersion, emulsification, homogenization, mixing, etc.) are spend in rotary pulse apparatus of cylindrical type which realise principles of discrete-pulsed input of energy.

Search of new equipment and technological decisions are directed on increase of an overall performance of devices and an intensification of processes in environments which requires theoretical researches on studying of hydrodynamic conditions, modelling of processes in new devices.

The purpose of the research is to study the changes of physical and chemical parameters of liquid binary systems: water and water-ethanol mixtures using reagent-free method of treatment, such as the influence of alternating impulses of pressure. 


\section{— Processes and Equipment of Food Productions -}

\section{Materials and methods}

\section{Materials}

Water and water-ethanol mixtures in a wide range of concentration were used for experiments. Percentage of ethanol in mixtures was varied from 5 to $90 \%$.

\section{Experimental installation}

This study was carried out in experimental form at the pilot unit. The main part of the pilot unit is a rotary pulsed apparatus in which liquids treat by alternating impulses of pressure [13].

\section{Sample preparation}

Water and water-ethanol mixtures were prepared using the method described by [14]. Water and ethanol of different types was used for mixing.

Water gave in to processing by alternating impulses of pressure before the technological process of receiving of mixtures. Water treatment and mixing process was spent in rotary pulse apparatus.

Liquid binary systems were passed through rotating coaxial cylinders with cuts on a surface and small clearances between them (which reach 100nm) instantly, that allowed to spend this process by continuous mode.

\section{Methods}

For the definition of physical and chemical parameters of liquid samples of binary systems which obtained during the experiment, standard methods described in special literature are used [15].

Study of quantity and quantitative changes of dissolved oxygen in water and waterethanol mixtures of different concentration from 5 to $90 \%$ is carried out with use microprocessor-based device oximeter EZODO PDO-408 with remote electrode.

Determination of change of potential of hydrogen and redox potential of liquid samples of binary systems is carried out with use analogue $\mathrm{pH}$-meter-millivoltmeter $\mathrm{pH}-150 \mathrm{M}$ with electrodes.

For receiving valid data, liquid samples were analyzed not less than three times with the following statistical processing.

\section{Results and discussion}

For research of changes of chemical and physical parameters of liquid binary systems such as technological water following properties were investigated:

- potential of hydrogen ( $\mathrm{pH}$ value);

- redox potential.

Influence of preliminary processing of water with application of method of discretepulsed input of energy for technology of receiving of water-ethanol mixtures was studied.

During processing of water and mixing of water and ethanol in the conditions of alternating impulses of pressure represented: 
- $\quad \Delta \mathrm{P}=370 \mathrm{kPa}$ near an external surface of an internal rotor;

- $\Delta \mathrm{P}=240 \mathrm{kPa}$ near an external surface stator;

- $\Delta \mathrm{P}=155 \mathrm{kPa}$ near an internal surface stator;

- $\Delta \mathrm{P}=190 \mathrm{kPa}$ near an internal surface of an external rotor.

Thus pressure of shift of a stream represented:

- $219,8 \mathrm{~Pa}$ (the first rotor);

- 235,5 Pa, (the second rotor)

Speeds of shift of a stream:

- $\quad 2,2 \times 10^{5} \mathrm{~s}^{-1}$ (the first rotor);

- $2,4 \times 10^{5} \mathrm{~s}^{-1}$ (the second rotor).

Linear speeds:

- $\quad 21,98 \mathrm{~m} / \mathrm{s}$ (the first rotor);

- $23,58 \mathrm{~m} / \mathrm{s}$ (the second rotor).

During water processing by alternating impulses of pressure the potential of hydrogen and reactionary ability of water varies. For carrying out of process of mixing of water and alcohol water gave in to treatment during special time from 30 s to 300 s.

The potential of hydrogen characterizes concentration of free ions of hydrogen in water and is one of the main working indicators of quality of water, in many respects defines character of chemical and biological processes which occur in water and water-ethanol mixtures. The change of $\mathrm{pH}$ value is shown in Table 1 .

Table 1

Change of potential of hydrogen under the alternating impulses of pressure treatment in water

\begin{tabular}{|c|c|c|c|c|c|}
\hline \multirow[t]{2}{*}{$\mathbf{N}$} & \multicolumn{2}{|c|}{ Potential of hydrogen, $\mathrm{pH}$} & \multicolumn{2}{|c|}{ Temterature, ${ }^{\circ} \mathrm{C}$} & \multirow{2}{*}{$\begin{array}{l}\text { Duration of } \\
\text { treatment, }\end{array}$} \\
\hline & $\begin{array}{c}\text { Before } \\
\text { treatment }\end{array}$ & $\begin{array}{c}\text { After } \\
\text { treatment }\end{array}$ & $\begin{array}{c}\text { Before } \\
\text { treatment }\end{array}$ & $\begin{array}{c}\text { After } \\
\text { treatment }\end{array}$ & \\
\hline 1 & 6,12 & 6,59 & 17,0 & 17,3 & 30 \\
\hline 2 & 6,09 & 6,60 & 16,3 & 16,9 & 60 \\
\hline 3 & 6,10 & 6,69 & 17,9 & 18,5 & 90 \\
\hline 4 & 6,09 & 6,75 & 18,0 & 19,2 & 120 \\
\hline 5 & 6,05 & 6,99 & 18,2 & 18,7 & 150 \\
\hline 6 & 6,13 & 7,18 & 17,5 & 18,3 & 180 \\
\hline 7 & 6,06 & 7,31 & 18,1 & 19,0 & 210 \\
\hline 8 & 6,12 & 7,48 & 17,9 & 18,8 & 240 \\
\hline 9 & 6,12 & 7,79 & 17,4 & 18,3 & 270 \\
\hline 10 & 6,10 & 7,99 & 18,0 & 18,9 & 300 \\
\hline
\end{tabular}

During researches increases $\mathrm{pH}$ of the distilled water on $13 \%$ have been established, thus the hydrogen potential of the water prepared on technology of the distillery has raised on $14-14,5 \%$.

Treatment of liquid binary systems such as water in the conditions of alternating impulses of pressure allows changing physical parameters throughout long time (6 month).

Value of redox potential $\mathrm{Eh}$ and $\mathrm{pH}$ are interdependent.

A direct potentiometry concerns also redoximetry. It is a measurement standard and real redox potentials and balance constants redox reactions. The redox potential depends from activity of oxidized form of substance. 


\section{— Processes and Equipment of Food Productions -}

In scientific work the presented researches concerning change redox potential in binary water systems.

The change of redox potential of water in technological process of mixing during experimental treatment in rotary pulse apparatus is shown in Table 2.

Table 2

Change of redox potential during experimental water treatment by alternating impulses of pressure

\begin{tabular}{|c|c|c|c|c|c|}
\hline \multirow{2}{*}{$\mathbf{N}$} & \multicolumn{2}{|c|}{ Redox potential, $\mathbf{~ m V}$} & \multicolumn{2}{c|}{ Temterature, ${ }^{\circ} \mathbf{C}$} & \multirow{2}{*}{$\begin{array}{c}\text { Duration of } \\
\text { treatment, } \mathbf{s}\end{array}$} \\
\cline { 2 - 5 } & $\begin{array}{c}\text { Before } \\
\text { treatment }\end{array}$ & $\begin{array}{c}\text { After } \\
\text { treatment }\end{array}$ & $\begin{array}{c}\text { Before } \\
\text { treatment }\end{array}$ & $\begin{array}{c}\text { After } \\
\text { treatment }\end{array}$ & \\
\hline 1 & +260 & +210 & 17,0 & 17,3 & 30 \\
\hline 2 & +264 & +211 & 16,3 & 16,9 & 60 \\
\hline 3 & +268 & +198 & 17,9 & 18,5 & 90 \\
\hline 4 & +270 & +132 & 18,0 & 19,2 & 120 \\
\hline 5 & +258 & +135 & 18,2 & 18,7 & 150 \\
\hline 6 & +258 & +132 & 17,5 & 18,3 & 180 \\
\hline 7 & +270 & +105 & 18,1 & 19,0 & 210 \\
\hline 8 & +273 & +101 & 17,9 & 18,8 & 240 \\
\hline 9 & +258 & +103 & 17,4 & 18,3 & 270 \\
\hline 10 & +271 & +110 & 18,0 & 18,9 & 300 \\
\hline
\end{tabular}

Results of researches are presented that redox potential of ware is value actable and at interaction with atmospheric air rises. In the isolated systems of increase occurs much more slowly.

A value of redox potential in the course of processing by alternating impulses of pressure depending on processing time decreases on $20-60 \%$.

For research of changes of chemical and physical parameters of liquid binary systems following properties were investigated:

- quantity of dissolved oxygen;

- potential of hydrogen ( $\mathrm{pH}$ value);

- redox potential.

The dissolved oxygen in water systems is in the form of molecules $\mathrm{O}_{2}$. Concentration of the dissolved oxygen in water systems is integrated value which is defined by a parity of different modes of physical and chemical, hydrodynamic processes which occur in water systems and on border of division of phases «water system-atmosphere».

Absorption of oxygen from atmosphere occurs on a surface of water systems. Speed of this process raises with temperature decrease, with increase of pressure and mineralization decrease.

The quantity of the dissolved oxygen is the significant parameter at processing of liquid systems.

The quantity of the dissolved oxygen influences on speed of oxidation-reduction reactions. The smaller quantity of the dissolved oxygen in water-ethanol mixtures, there is more long their storage time. The change of quantity of dissolved oxygen by the alternating impulses of pressure treatment in water-ethanol mixtures is shown in Table 3. 
Table 3

Change of quantity of dissolved oxygen by the alternating impulses of pressure treatment in water-ethanol mixtures

\begin{tabular}{|c|c|c|c|c|c|}
\hline \multirow{2}{*}{$\mathbf{N}$} & \multicolumn{2}{|c|}{$\begin{array}{c}\text { Quantity of dissolved } \\
\text { oxygen, mg/l }\end{array}$} & \multicolumn{2}{|c|}{ Temterature, ${ }^{\circ} \mathbf{C}$} & \multirow{2}{*}{$\begin{array}{c}\text { Percentage of } \\
\text { ethanol, } \%\end{array}$} \\
\cline { 2 - 4 } & $\begin{array}{c}\text { Before } \\
\text { treatment }\end{array}$ & $\begin{array}{c}\text { After } \\
\text { treatment }\end{array}$ & $\begin{array}{c}\text { Before } \\
\text { treatment }\end{array}$ & $\begin{array}{c}\text { After } \\
\text { treatment }\end{array}$ & \\
\hline 1 & 11,1 & 5,6 & 14,0 & 14,3 & 5 \\
\hline 2 & 10,9 & 5,5 & 14,3 & 14,4 & 10 \\
\hline 3 & 10,8 & 5,8 & 14,3 & 14,5 & 15 \\
\hline 4 & 10,2 & 5,1 & 14,7 & 14,7 & 20 \\
\hline 5 & 10,4 & 5,8 & 14,5 & 14,7 & 25 \\
\hline 6 & 10,1 & 5,3 & 14,7 & 14,9 & 30 \\
\hline 7 & 10,2 & 5,3 & 14,4 & 14,5 & 35 \\
\hline 8 & 10,1 & 5,2 & 14,6 & 14,9 & 40 \\
\hline 9 & 10,9 & 6,0 & 14,2 & 14,6 & 45 \\
\hline 10 & 10,8 & 5,9 & 14,3 & 14,7 & 50 \\
\hline 11 & 11,0 & 5,5 & 14,2 & 14,4 & 55 \\
\hline 12 & 10,1 & 5,5 & 14,6 & 15,0 & 60 \\
\hline 13 & 10,2 & 5,5 & 14,5 & 14,9 & 65 \\
\hline 14 & 10,8 & 5,4 & 14,3 & 14,7 & 70 \\
\hline 15 & 11,5 & 6,3 & 14,6 & 15,1 & 75 \\
\hline 16 & 11,7 & 5,9 & 14,7 & 15,2 & 80 \\
\hline 17 & 10,9 & 5,5 & 14,3 & 14,9 & 85 \\
\hline 18 & 10,5 & 5,3 & 14,5 & 15,2 & 90 \\
\hline
\end{tabular}

Besides, it is established that at influence of alternating impulses of pressure in liquid binary systems such as water systems and water-ethanol mixtures occurs intensive deoxygenating, decrease in quantity of the dissolved oxygen in water and water-ethanol mixtures in comparison with the initial maintenance occurs practically on 50-55\%.

Owing to deoxygenating in aqueous-alcoholic mixes the quantity of harmful impurities, owing to decrease in reactionary ability of free radicals decreases. Such results confirm that carrying out of process of mixing in the conditions of alternating impulses of pressure is capable to slow down oxidizing reactions in water-ethanol mixtures. It is very important condition and it gives additional possibilities for producing of safe alcoholic beverages.

For technological process of receiving of alcoholic beverages and alcoholic products the great value has change of a $\mathrm{pH}$ value to initial value to processing.

Depending $\mathrm{pH}$ value on speed of course of chemical reactions can change.

The change of potential of hydrogen in conditions of the alternating impulses of pressure during mixing water and ethanol is shown in a Table 4 . 
Table 4

Change of potential of hydrogen in conditions of the alternating impulses of pressure in waterethanol mixtures

\begin{tabular}{|c|c|c|c|c|c|}
\hline \multirow[t]{2}{*}{$\mathbf{N}$} & \multicolumn{2}{|c|}{ Potential of hydrogen, pH } & \multicolumn{2}{|c|}{ Temterature, ${ }^{0} \mathrm{C}$} & \multirow{2}{*}{$\begin{array}{c}\text { Percentage of } \\
\text { ethanol, } \%\end{array}$} \\
\hline & $\begin{array}{c}\text { Before } \\
\text { treatment }\end{array}$ & $\begin{array}{c}\text { After } \\
\text { treatment }\end{array}$ & $\begin{array}{c}\text { Before } \\
\text { treatment }\end{array}$ & $\begin{array}{c}\text { After } \\
\text { treatment }\end{array}$ & \\
\hline 1 & 6,79 & 7,90 & 14,0 & 14,3 & 5 \\
\hline 2 & 6,81 & 7,92 & 14,3 & 14,4 & 10 \\
\hline 3 & 6,77 & 7,96 & 14,3 & 14,5 & 15 \\
\hline 4 & 6,80 & 7,90 & 14,7 & 14,7 & 20 \\
\hline 5 & 6,80 & 8,01 & 14,5 & 14,7 & 25 \\
\hline 6 & 6,75 & 7,93 & 14,7 & 14,9 & 30 \\
\hline 7 & 6,80 & 7,92 & 14,4 & 14,5 & 35 \\
\hline 8 & 6,79 & 7,97 & 14,6 & 14,9 & 40 \\
\hline 9 & 6,84 & 8,01 & 14,2 & 14,6 & 45 \\
\hline 10 & 6,79 & 7,92 & 14,3 & 14,7 & 50 \\
\hline 11 & 6,77 & 7,91 & 14,2 & 14,4 & 55 \\
\hline 12 & 6,79 & 7,92 & 14,6 & 15,0 & 60 \\
\hline 13 & 6,77 & 7,91 & 14,5 & 14,9 & 65 \\
\hline 14 & 6,78 & 7,92 & 14,3 & 14,7 & 70 \\
\hline 15 & 6,75 & 7,96 & 14,6 & 15,1 & 75 \\
\hline 16 & 6,78 & 7,92 & 14,7 & 15,2 & 80 \\
\hline 17 & 6,82 & 8,00 & 14,3 & 14,9 & 85 \\
\hline 18 & 6,81 & 7,97 & 14,5 & 15,2 & 90 \\
\hline
\end{tabular}

Application of alternating impulses of pressure in technology of receiving of alcoholic mixtures allows receiving the activated water with the certain physical properties and parameters, assured value of a $\mathrm{pH}$.

As a result of the carried out researches change of physical and chemical properties and parameters of water systems has been established at processing application high-frequency oscillation which it is possible to explain change of reactionary ability, owing to initiation of carrying over of a proton in associated liquids such as water, aliphatic alcohols, waterethanol mixtures with different percentage of ethanol and formation of a grid of hydrogen bonds which in turn influences the structural organisation and a structure.

The change of redox potential during mixing water and ethanol by alternating impulses of pressure is shown in a Table 5 .

During carrying out of experimental investigations carrying out of researches of systems in which water for mixing have been realised was not exposed to activation, was exposed to activation, and processing of a mix which has been received by distillery technology in the tanks with a mixer without additional activation of water was carried out.

A value of redox potential in the course of processing by alternating impulses of pressure in water-ethanol mixtures depending on processing time.

Carrying out of process of mixing is direct in a continuous mode and water treatment before mixing process give possibility to decrease redox potential on $22-63 \%$.

The general tendency to decrease redox which takes place during processing on an extent 120 s has been noted. Figure 1 after that there is not substantial increase redox. 
Table 5

Change of redox popential during mixing water and ethanol by alternating impulses of pressure

\begin{tabular}{|c|c|c|c|c|c|}
\hline \multirow[t]{2}{*}{$\mathbf{N}$} & \multicolumn{2}{|c|}{ Redox potential, $\mathrm{mV}$} & \multicolumn{2}{|c|}{ Temterature, ${ }^{0} \mathrm{C}$} & \multirow{2}{*}{$\begin{array}{c}\text { Percentage } \\
\text { of ethanol, } \\
\%\end{array}$} \\
\hline & $\begin{array}{c}\text { Before } \\
\text { treatment }\end{array}$ & $\begin{array}{c}\text { After } \\
\text { treatment }\end{array}$ & $\begin{array}{c}\text { Before } \\
\text { treatment }\end{array}$ & $\begin{array}{c}\text { After } \\
\text { treatment }\end{array}$ & \\
\hline 1 & +115 & +82 & 14,0 & 14,3 & 5 \\
\hline 2 & +120 & +86 & 14,3 & 14,4 & 10 \\
\hline 3 & +121 & +85 & 14,3 & 14,5 & 15 \\
\hline 4 & +119 & +84 & 14,7 & 14,7 & 20 \\
\hline 5 & +120 & +84 & 14,5 & 14,7 & 25 \\
\hline 6 & +115 & +76 & 14,7 & 14,9 & 30 \\
\hline 7 & +117 & +70 & 14,4 & 14,5 & 35 \\
\hline 8 & +118 & +70 & 14,6 & 14,9 & 40 \\
\hline 9 & +117 & +75 & 14,2 & 14,6 & 45 \\
\hline 10 & +120 & +77 & 14,3 & 14,7 & 50 \\
\hline 11 & +121 & +78 & 14,2 & 14,4 & 55 \\
\hline 12 & +122 & +82 & 14,6 & 15,0 & 60 \\
\hline 13 & +119 & +85 & 14,5 & 14,9 & 65 \\
\hline 14 & +120 & +85 & 14,3 & 14,7 & 70 \\
\hline 15 & +121 & +88 & 14,6 & 15,1 & 75 \\
\hline 16 & +117 & $\begin{array}{r}+83 \\
\end{array}$ & 14,7 & 15,2 & 80 \\
\hline 17 & +115 & +86 & 14,3 & 14,9 & 85 \\
\hline 18 & +116 & +86 & 14,5 & 15,2 & 90 \\
\hline
\end{tabular}

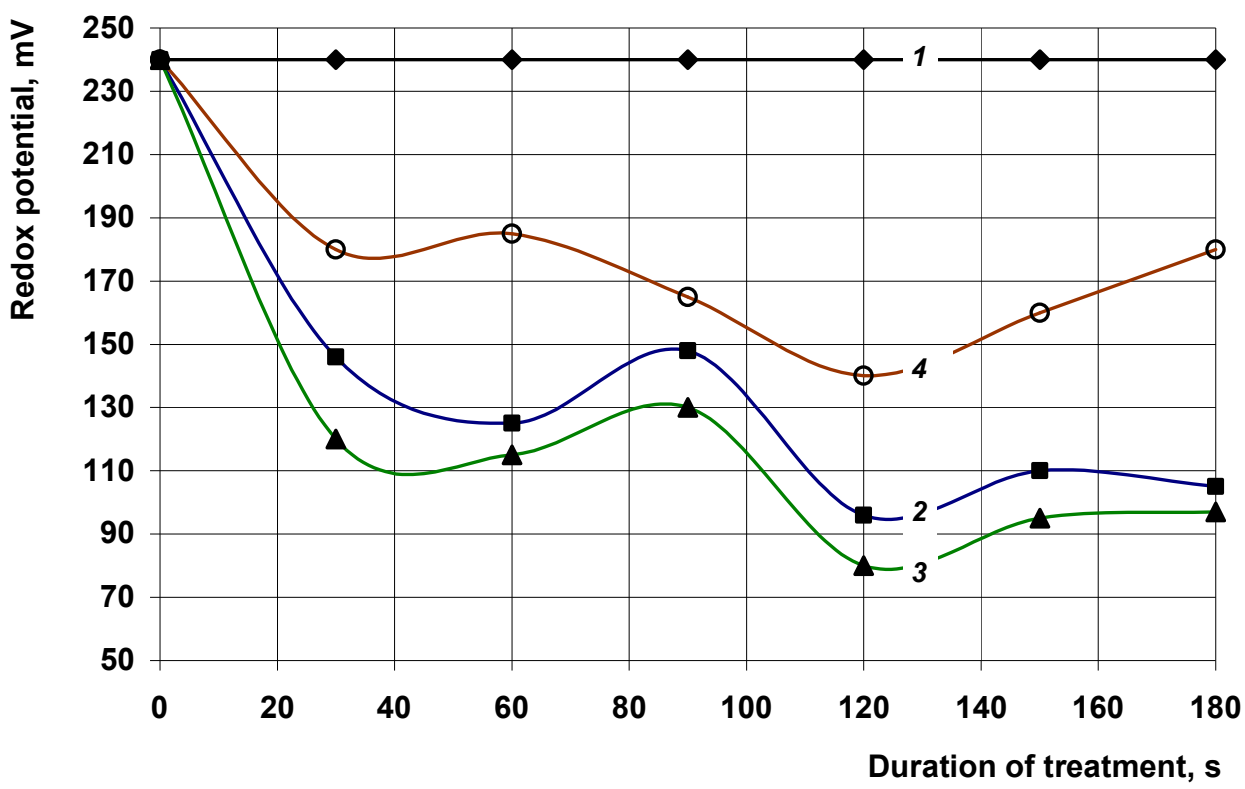

\footnotetext{
$\neg 1$ Water-ethanol mixture without treatment

$\rightarrow 2$ Water-ethanol mixture with non treatment water

-3 Water-ethanol mixture with treatment water

- 4 Water-ethanol mixture after treatment
}

Figure 1. Profile changes redox during mixing process 


\section{— Processes and Equipment of Food Productions -}

The obtained data confirm, that the lowest level of redox was observed in waterethanol mixtures which have been received with application of alternating impulses of pressure. Also it is necessary to pay attention that water in such mixtures gave in to processing in the conditions of alternating impulses of pressure. The general level of decrease redox in comparison with the initial makes $65 \%$.

\section{Conclusions}

As a result of research, it was found that the water and water-ethanol mixtures treatment by nonchemical reagentless method of discrete-pulsed input of energy in rotary pulse devices can greatly reduce the duration of the process of mixing mode, reduce power consumption, increase capacity and replace the batch process for the continuous mixing.

Experimental and theoretical studies have shown that rotary pulse apparatus may be suitable for processing in food industry, where hydrodynamic effects are found to be an alternative to traditional mixing tanks.

A detailed analysis of experimental data showed that the use of alternating impulses of pressure in the preparation of water-ethanol mixtures in a wide range of concentration allows obtaining mixtures with improved physical and chemical parameters.

\section{References}

1. Mijaković M. Kežić B., Zoranić L., Sokolić F., Asenbaum A., Pruner C., Emmerich Wilhelm, Perera A. (2011), Ethanol-water mixtures: ultrasonics, Brillouin scattering and molecular dynamics, Journal of Molecular Liquids, 164(1-2), pp 66-73.

2. Marcin Rybicki, Ewa Hawlicka (2014), Influence of ions on molecular vibrations and hydrogen bonds in methanol-water mixtures: MD simulation study, Journal of Molecular Liquids, 196, pp 300-307.

3. Doosti M.R., Kargar R., Sayadi M.H. (2012), Water treatment using ultrasonic assistance: A review, Proceedings of the International Academy of Ecology and Environmental Sciences, 2(2), pp. 96-110.

4. Mahvi A.H. (2009), Application of ultrasonic technology for water and wastewater treatment, Iranian J Publ Health, 38(2), pp.1-17.

5. Yamashita M., Duffield C., Tiller W.A. (2003), Langmuir, 19, pp. 6851-6856.

6. Laughrey Z., Bear E., Jones R., Tarr M.A. (2001), Aqueous sonolytic decomposition of polycyclic aromatic hydrocarbons in the presence of additional dissolved species, UltrasonSonochem, 8, pp. 353-357.

7. Philippe Vallee, Jacques Lafait, Laurent Legrand, Pascale Mentre, MarieOdile.Monod, Yolene Thomas (2005), Effects of pulsed low frequency electromagnetic fields on water characterized by light scattering techniques: role of bubbles, Langmuir, pp. 1-17.

8. Oshitani, J.; Uehara, R.; Higashitani, K. (1999) Low frequency electromagnetic fields J. Colloid Interface Sci., 209, 374-379.

9. Chua S.Y., Adul Latif P., Ibrahim Sh., et al. (2010), Effect of ultrasonic irradiation on COD and TSS in raw rubber mill effluent,Environment Asia, 3(special issue), pp. 3235.

10. Jiang Y., Petrier C.H., Waite T.D. (2002), Effect of $\mathrm{pH}$ on the ultrasonic degradation of ionic aromatic compounds in aqueos solution, Ultrason Sonochem, 9, pp. 163-168. 
11. Sarah Ede (2006), Infrared and photocatalytic studies of model bacterial species for water treatment, Inorganic Materials Research Program Sch. of Phys. and Chem. Sciences Queensland Univ. of Technology, pp. 9-11.

12. Dolinskij A.A., Basok B.I. (2005), Nanoscale effects by discrete-pulsed transformation of energy, IFZH, 78(1), pp. 15-23.

13. Dubovkina I.O. (2015), Features of carrying out of process of mixing of water and spirit in the conditions of sign-variable impulses of pressure, Technology audit and production reserves, $6 / 1(26)$, pp. 42-45

14. Shurchkova Ju., Dubovkina I. (2015), Research parameters of the water-ethanol mixture obtained under conditions of alternating impulses of pressure, Bulletin of NTU "KhPI". Series: New solutions in modern technologies, 46(1155), pp. 171-176.

15. Kenneth I. Ozomwna (2007), Recent Advances in Analitycal Electrochemistry, Transword Research in Analitycal Electrochemistry, Available at:

Network.http://www.ressign.com/UserBookDetail.aspx?bkid=605\&catid=160 